\title{
O Modelo de Jo Mynard Para o Aconselhamento Linguageiro Como Propulsor da Aquisição de Segunda Língua
}

\section{Jo Mynard's Model for Advising in Language Learning as a Propeller of Second Language Acquisition}

DOI: $10.46814 / 1 \mathrm{ajdv} 3 \mathrm{n} 4-055$

Recebimento dos originais: 01/05/2021

Aceitação para publicação: 31/06/2021

\section{Isabelly Raiane Silva dos Santos}

Mestranda em Letras pelo Programa de Pós-graduação em Letras (PPGL) da Universidade Federal do Pará (UFPA)

Docente no Instituto Federal de Educação, Ciência e Tecnologia do Pará (IFPA)

Endereço institucional: Rodovia Ernesto Acyoli, S/N, Km 3 - Nova Colina, Altamira-PA, Brasil

E-mail: isabelly.santos@ifpa.edu.br

\section{RESUMO}

$\mathrm{O}$ aconselhamento linguageiro pode ser definido como uma prática a qual busca encorajar os aprendentes a serem mais conscientes acerca de seus processos de aprendizagem a fim de torná-los mais autônomos. Nesse sentido, o objetivo desta pesquisa é descrever a relação que a prática eficaz do aconselhamento linguageiro estabelece com a aprendizagem bem-sucedida de línguas estrangeiras. Tomando como embasamento teórico conceitos e definições propostas por Gardner e Miller (1999), Benson (2001), Reinders (2008), Carson e Mynard (2012) e Aoki (2012), foram identificados aspectos relacionados à promoção da autonomia e à manutenção da motivação, visto que esses são conceitos interdependentes os quais auxiliam na descoberta do caminho para sucesso da aprendizagem. Com a finalidade de consolidar esta pesquisa de cunho qualitativo, foram analisadas as atitudes com relação à aprendizagem tomadas por uma estudante do curso de graduação em Licenciatura em Letras com habilitação em Inglês a partir do momento no qual ela procurou a ajuda do aconselhamento linguageiro para aprender a aprender. Para tanto, os instrumentos de pesquisa utilizados incluem questionários, entrevistas, narrativa de aprendizagem, ficha de metas pessoais, relatos orais e desenhos. A partir disso, foram observadas diversas mudanças na trajetória de aprendizagem da aluna, que aos poucos pôde se autocompreender e entender o funcionamento de sua própria cognição. Desse modo, os resultados da investigação apontaram a prática do aconselhamento linguageiro como algo favorável e benéfico ao estabelecimento da autonomia e da motivação estudantil.

Palavras-chave: Aconselhamento linguageiro. Aprendizagem. Aquisição de segunda língua.

\begin{abstract}
Advising in language learning can be defined as a practice which seeks to encourage learners to be more aware of their learning processes in order to make them more autonomous. In this sense, the objective of this research is to describe the relationship that the effective practice of advising in language learning establishes with the successful learning of foreign languages. Taking as theoretical basis concepts and definitions proposed by Gardner and Miller (1999), Benson (2001), Reinders (2008), Carson and Mynard (2012) and Aoki (2012), aspects related to the promotion of autonomy and the maintenance of motivation were identified, as they are interdependent concepts which help to discover the path to learning success. In order to consolidate this qualitative research, the attitudes towards learning taken by an undergraduate student Majoring in English Language, from the moment she sought the help of advising in language learning to learn, were analyzed. For that, the research
\end{abstract}


instruments used include questionnaires, interviews, learning narrative, personal goals sheet, oral reports and drawings. From this, several changes were observed in the student's learning trajectory, who gradually was able to self-understand and understand the functioning of her own cognition. Thus, the results of the investigation pointed to the practice of advising in language learning as something favorable and beneficial to the establishment of student autonomy and motivation.

Keywords: Advising in language learning. Learning. Second language aquisition.

\section{INTRODUÇÃO}

Estudar uma língua estrangeira envolve inúmeros aspectos, dentre eles: vontade, esforço e persistência. É enorme a quantidade de obstáculos e empecilhos que rondam a aprendizagem. Além do mais, ainda é necessário alcançar um bom nível de proficiência no idioma para que um profissional se torne capacitado para atuar na área. Em outras palavras, estudar uma língua estrangeira é um processo longo durante o qual pode haver desmotivação e/ou desistência.

Partindo do princípio de que o aprendente necessita de um incentivo para continuar sua trajetória de aprendizagem, o aconselhamento linguageiro surge como um tipo de suporte. Neste processo, os conselheiros se encontram com os aconselhados de forma individual para oferecer feedback e conselhos relacionados à aprendizagem (REINDERS, 2008).

O principal objetivo dessa prática é ajudar os alunos a desenvolverem habilidades de aprendizagem autodirigida, fazendo com que se tornem aprendentes autônomos. Nesse contexto, o conselheiro concentra sua prática nas necessidades individuais de cada discente, personaliza as atividades e, assim, estudantes são encorajados a refletirem sobre sua própria aprendizagem.

Para isso, é necessário que seja instaurado um ambiente propício à prática do aconselhamento. Um dos pontos que devem ser levados em consideração na instauração desse ambiente é a criação de uma relação empática entre os agentes, fazendo com que o aprendente possa relatar experiências difíceis com a língua estrangeira para que, futuramente, seja auxiliado pelo conselheiro.

Partindo desses argumentos, o objetivo geral desta pesquisa é descrever a relação que a prática eficaz do aconselhamento linguageiro estabelece com a aprendizagem bem-sucedida de línguas estrangeiras. Dentre os objetivos específicos, é possível mencionar: descrever o sistema de aprendizagem de uma estudante de inglês e identificar aspectos que evidenciem o benefício do apoio do conselheiro para a aprendizagem autônoma.

Assim, para guiar a investigação, foram formuladas as seguintes perguntas de pesquisa: 1) Qual é a relação que a prática eficaz do aconselhamento linguageiro estabelece com a aprendizagem bemsucedida de línguas estrangeiras? 2) Como pode ser descrito o sistema de aprendizagem de uma 
estudante de inglês? 3) Quais aspectos evidenciam o benefício do apoio do conselheiro para a aprendizagem autônoma?

Além desta introdução, este artigo está dividido do seguinte modo: na próxima seção, são discutidos aspectos relacionados ao referencial teórico. Para tanto, foram utilizados conceitos de autores brasileiros e estrangeiros. Em seguida, a metodologia adotada no processo investigativo é detalhada. Após isso, o resultado da análise do corpus e a discussão de dados é realizada. Seguidamente, as conclusões emergidas a partir da realização da pesquisa são expostas. Ao final, as referências bibliográficas consultadas são listadas.

\section{REFERENCIAL TEÓRICO}

Esta seção aborda aspectos relevantes referentes à base teórica deste estudo. Ela está dividida em duas subseções. Na primeira, são destacadas as definições propostas para o conceito de aconselhamento linguageiro, assim como são apresentadas as características dessa prática. Na segunda, o modelo da autora Jo Mynard para o aconselhamento linguageiro é descrito e analisado.

\subsection{ACONSELHAMENTO LINGUAGEIRO}

Mozzon-McPherson (1997) afirma que o aconselhamento linguageiro é uma atividade de natureza colaborativa a qual envolve funções ativas, reativas e interativas. Do mesmo modo, para Carson e Mynard (2012), é um processo em que se oferece ajuda aos alunos com a intenção de direcioná-los para seus próprios caminhos. De maneira semelhante, Esch (1996) acredita que o aconselhamento consiste em um sistema de intervenção que objetiva dar suporte à metodologia de aprendizagem de língua dos alunos utilizando-se, para isso, de conversas. Seguindo a mesma linha de raciocínio, conforme Reinders (2008), o aconselhamento linguageiro é um tipo de suporte de língua em que os professores se encontram com os alunos de forma individual para oferecer conselhos e feedback a fim de ajudá-los a desenvolverem habilidades de aprendizagem autodirigida.

Um aconselhamento linguageiro eficaz se caracteriza pela ação do conselheiro em trabalhar com o aprendente de forma individualizada, concentrar sua prática nas necessidades individuais do aluno, personalizar as atividades e encorajar os discentes a refletirem sobre sua aprendizagem, fazendo com que se tornem aprendentes autônomos.

Apesar de apresentarem aspectos e funções divergentes, é muito comum que um conselheiro seja confundido com um professor. Um dos pontos o qual faz com que esse argumento ganhe mais força é que ambos lidam com o aprendente e o colocam como elemento-chave do ensino/aprendizagem. Nesse sentido, a Tabela 1 tem como objetivo desmitificar a ideia equivocada de igualdade entre as funções assumidas por professores e conselheiros linguageiros. 
Tabela 1: Diferenças entre professor e conselheiro

\begin{tabular}{|c|c|}
\hline PROFESSORES & CONSELHEIROS \\
\hline Professores são vistos como líderes. & Conselheiros são vistos como colaboradores. \\
\hline $\begin{array}{l}\text { Geralmente há um currículo/ementa pré- } \\
\text { determinado. }\end{array}$ & Há uma negociação dos conteúdos trabalhados. \\
\hline Professores atuam como avaliadores dos alunos. & $\begin{array}{l}\text { Conselheiros discutem com os alunos diferentes maneiras } \\
\text { de auto monitorar o seu progresso. }\end{array}$ \\
\hline Professores são instrutores/organizadores. & Conselheiros são ouvintes reflexivos. \\
\hline $\begin{array}{l}\text { Professores usam uma variedade de material } \\
\text { didático. }\end{array}$ & $\begin{array}{l}\text { Conselheiros demonstram aos aprendentes como usar } \\
\text { materiais e equipamentos. }\end{array}$ \\
\hline $\begin{array}{l}\text { Professores monitoram uma turma inteira e } \\
\text { procuram problemas em comum. }\end{array}$ & $\begin{array}{l}\text { Conselheiros discutem problemas individuais de } \\
\text { determinado aprendente. }\end{array}$ \\
\hline $\begin{array}{l}\text { Professores fornecem feedback para as tarefas } \\
\text { propostas. }\end{array}$ & $\begin{array}{l}\text { Conselheiros encorajam os alunos a refletir sobre os } \\
\text { resultados obtidos nas tarefas. }\end{array}$ \\
\hline
\end{tabular}

Fonte: Gardner e Miller (1999, p. 182).

\subsection{O MODELO DE JO MYNARD PARA O ACONSELHAMENTO LINGUAGEIRO}

De acordo com a localidade, a cultura, as práticas e os costumes de um povo, o aconselhamento linguageiro pode ocorrer de distintos modos: em grupo ou em pares, online ou dentro da própria sala de aula, como parte de uma disciplina, entre outros.

Um dos modelos mais famosos e mais bem-sucedidos para essa prática foi proposto por Mynard (2012), e atualmente é o mais adotado nas universidades referências na concessão do serviço de aconselhamento linguageiro, como a Universidade Kanda, no Japão, e a Universidade de Hull, na Ingraterra. Este modelo tem como foco três pontos primordiais: o diálogo, as ferramentas e o contexto.

O diálogo expressa a interação entre os agentes envolvidos. Por isso, pode ser considerado o elemento central e norteador dessa prática. Além disso, há também o diálogo interno, no qual ambos os agentes formulam suas ideias antes de expô-las.

Para Kato (2012), o diálogo intencionado encoraja os conselheiros a se engajarem em um nível mais profundo de aprendizagem transformativa, vivenciando valores existentes e identificando discrepâncias entre suas crenças e seu real comportamento.

No que diz respeito às ferramentas, elas se dividem em cognitivas, como, por exemplo, folhas de autoavaliação e planos de aprendizagem; teóricas, as quais são conhecimentos acerca de crenças e estratégias para a aprendizagem; e ferramentas práticas, dentre as quais é possível citar o gravador de áudio e os locais reservados para a condução das sessões de aconselhamento. 


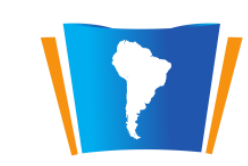

LATIN AMERICAN

Em relação ao terceiro pilar do modelo de aconselhamento proposto por Mynard (2012), o contexto pode se caracterizar como físico, ou seja, o ambiente no qual a sessão ocorrerá; pessoal, que varia de acordo com os interesses dos agentes; e práticas contextuais, as quais envolvem relações variadas com comunidades de prática.

Além do mais, o aconselhado exerce um papel crucial neste processo, visto que se ele não expressar suas ideias, o aconselhamento jamais ocorrerá. Por conseguinte, é dada mais atenção a ele que ao conselheiro, como ilustra a "Figura 1".

Figura 1: O modelo de Mynard (2012) para o aconselhamento linguageiro

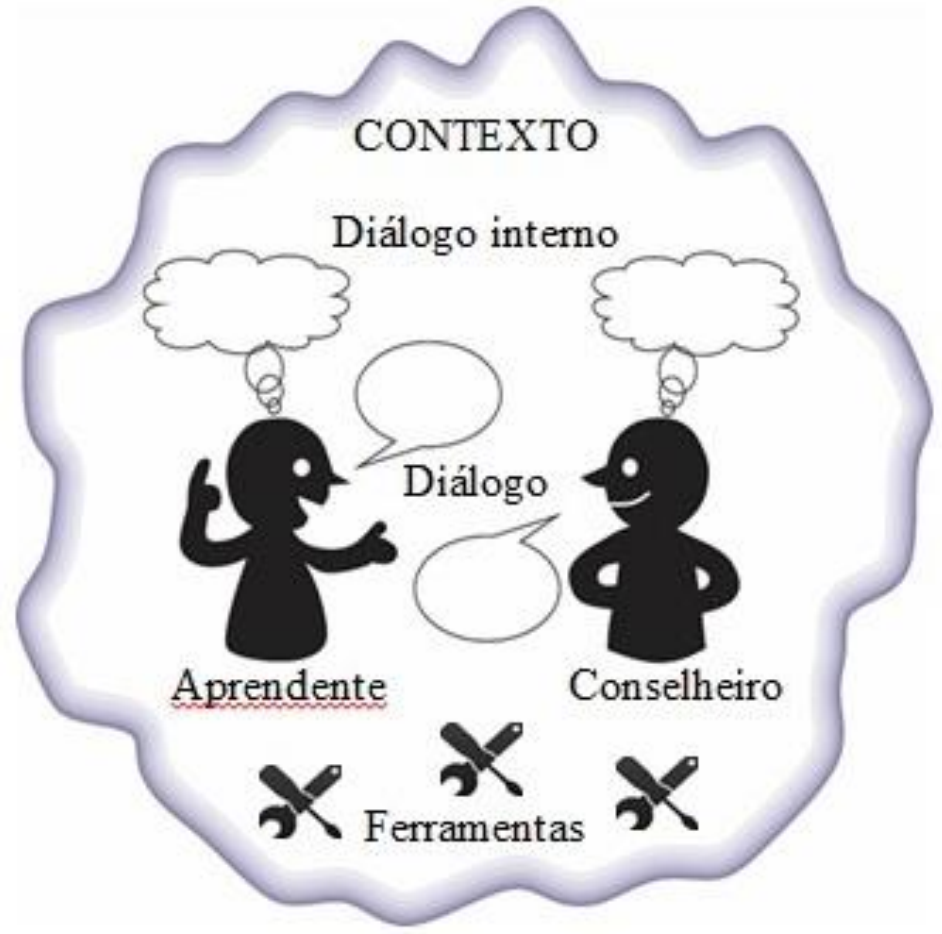

Fonte: Mynard (2012, p. 33).

Em relação à figura acima, observa-se que o aconselhamento não é uma técnica estática e instrumentalista que pode ser aprendida e aplicada. De modo contrário, o desenvolvimento do aconselhamento linguageiro precisa ser um processo contínuo (GARDNER; MILLER, 1999). Portanto, não importa o quão experiente seja um conselheiro, treinamento contínuo precisa ser fornecido a fim de assegurar o desenvolvimento profissional constante (KATO, 2012).

\section{METODOLOGIA}

Com a finalidade de consolidar esta pesquisa de caráter qualitativo, foram analisadas as atitudes com relação à aprendizagem tomadas por uma estudante do primeiro semestre do curso de graduação em Licenciatura em Letras com habilitação em Inglês a partir do momento no qual procurou ajuda do 
aconselhamento linguageiro para aprender a aprender, visto que sua trajetória de aprendizagem encontrava empecilhos e barreiras as quais impossibilitavam a aprendizagem eficaz do idioma estrangeiro.

Ao longo de quatro meses foram estabelecidas seis sessões de aconselhamento linguageiro presenciais e online, por meio de um aplicativo de troca de mensagens. Grande parte das sessões presenciais ocorreu na parte externa do Instituto de Letras e Comunicação (ILC) na Universidade Federal do Pará (UFPA), instituição onde a participante da pesquisa cursava o curso de graduação.

Para coletar dados, os instrumentos de pesquisa utilizados longo das sessões de aconselhamento linguageiro foram: questionários, entrevistas, narrativa de aprendizagem, ficha de metas pessoais, relatos orais e desenhos produzidos pela aconselhada.

Os dados coletados foram organizados pela autora deste estudo, que possuía um diário de campo para realizar anotações. Essas continham as interpretações dos fatos ocorridos nos encontros e as transcrições de depoimentos relatados pela participante da pesquisa, a qual é identificada pelo código AC5CL22. Com base nisso, a análise das informações foi feita a partir da comparação entre os achados da pesquisa e a teoria proposta por autores referência neste campo de estudo.

\section{RESULTADOS E DISCUSSÃO}

A aconselhada, identificada pelo código AC5CL22, era caloura do curso de graduação em Letras com habilitação em Inglês, e demonstrava proficiência no idioma. Ela estudou inglês em um curso de idiomas privado e o concluiu com sucesso, o que a possibilitou se comunicar no idioma-alvo.

AC5CL22 procurou a ajuda do aconselhamento, pois possuía dificuldades em relação à habilidade de compreensão oral (listening). Em outras palavras, nem sempre conseguia compreender as frases devido à velocidade com a qual as pessoas falavam em inglês.

Outro aspecto que precisou ser trabalhado foi a habilidade de produção oral (speaking). Segundo os relatos, a estudante sabia como dizer as palavras em inglês, mas não conseguia encontrar o contexto correto para aplicá-las. Ademais, sentia vergonha ao falar em público e cometia erros relacionados à pronúncia e à gramática.

No que diz respeito à utilização de materiais didáticos para fomentar a aprendizagem, o grande acervo de material que possuía garantiu à aconselhada a energia necessária para tomar iniciativas rumo ao alcance de seu objetivo: desenvolver a oralidade em língua inglesa de forma coerente.

Desde o início do processo, foi estabelecida uma relação amigável e respeitosa entre aconselhada e conselheira, a autora desde estudo. Nessa perspectiva, a empatia no processo de aconselhamento possui grande respaldo pela literatura da área (AOKI, 2012; MOZZON-MC PHERSON, 2007). Este nível de importância dado ao aconselhamento pode ser fundamental para 
garantir o sucesso no cumprimento de metas e, consequentemente, o progresso na trajetória dos participantes.

AC5CL22 também se beneficiou do uso de estratégias metacognitivas as quais, de acordo com Oxford (1990), são aquelas que auxiliam no processo de coordenação da aprendizagem.

No decorrer da condução das sessões de aconselhamento linguageiro, a aluna conseguiu organizar seus estudos. Ela entregou todas as tarefas no prazo estipulado e conseguiu seguir, de modo estável, uma rotina de estudos. O uso da agenda como instrumento de controle foi algo constante durante todo o período. Essa prática resultou em sua aprovação com ótimos conceitos em todas as disciplinas da graduação.

Outro ponto fundamental foi a identificação de iniciativas autônomas por parte da aconselhada. Seguindo a noção de autonomia proposta por Benson (2001), definida como a capacidade de tomar controle ou responsabilidade por sua própria aprendizagem, foi possível notar que, após as sessões iniciais, AC5CL22 já conseguia estabelecer objetivos, planejar suas ações e agir por conta própria. A “Tabela 2" ilustra a ficha de metas da aconselhada. De modo positivo, todas as metas estabelecidas por ela foram cumpridas de forma virtuosa.

Tabela 2: Ficha de metas da aconselhada

\begin{tabular}{|c|c|c|}
\hline 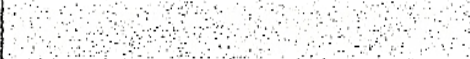 & \multicolumn{2}{|l|}{ Ficha de Aconselhamento Linguageiro } \\
\hline $\begin{array}{l}\text { METAS PESSOAIS } \\
\text { o que gostaria de... }\end{array}$ & $\begin{array}{l}\text { SUGESTÕES/COMPROMETIMENTO } \\
\text { O que devo fazer. }\end{array}$ & $\begin{array}{l}\text { PROGRESSO } \\
\text { Como foil meu } \\
\text { desempenho no período }\end{array}$ \\
\hline $\begin{array}{l}\text { Ver um filme complets } \\
\text { lou sp de sériel andender } \\
\text { óudis }\end{array}$ & $\begin{array}{l}\text { Treimar o listening paro poder } \\
\text { entender a cúdia em irglés. }\end{array}$ & $-1--2--3--4-\infty$ \\
\hline $\begin{array}{l}\text { Deacobrir corno algumas } \\
\text { polar ron do dia-a - dia sos } \\
\text { ditas em ingles. }\end{array}$ & Verifion o dicionório mais veges & $-2---3--4--5$ \\
\hline $\begin{array}{l}\text { Ler um livro complets } \\
\text { em ingles. }\end{array}$ & $\begin{array}{l}\text { Goston menos tempes snos redes } \\
\text { sociais e vendo moticios mo inteunit. }\end{array}$ & $--1--2---3---4--5$ \\
\hline $\begin{array}{l}\text { Escuton o audio do livo } \\
\text { O Homem Elephante }\end{array}$ & $\begin{array}{l}\text { Bedicar un tempo no final de } \\
\text { semana para cumprir a tarefa. } \\
\text { Escutor menas música. }\end{array}$ & $---1--2--3---4---5$ \\
\hline
\end{tabular}

Fonte: a autora. 
Dois meses após o início do aconselhamento, foi possível identificar altos níveis de motivação na aluna, o que possibilitou a experimentação de uma aprendizagem autônoma: ela procurava novos modos de aprender. Portanto, resultados apontam para a conscientização sobre a aprendizagem da língua. Assim, a responsabilidade foi recaída sob a aprendente, e isso contribuiu para a instauração de uma aprendizagem ativa.

A "Figura 2" representa a ideia que a aconselhada tinha de si no presente e no futuro, dez anos após o momento em que ela produziu o desenho. Por conseguinte, é possível verificar a ilustração da mesma pessoa em duas fases da vida. Na primeira parte da imagem, que representa o presente, a aconselhada é aluna da graduação e utiliza ferramentas como livro didático, fone de ouvido, caderno e dicionário para impulsionar a aprendizagem. Na parte direita da imagem, é evidenciado seu papel como professora no futuro, dez anos após o desenho ter sido feito. Segundo os relatos de AC5CL22, ela gostaria de aparentar uma postura séria e comprometida com a aprendizagem de seus alunos.

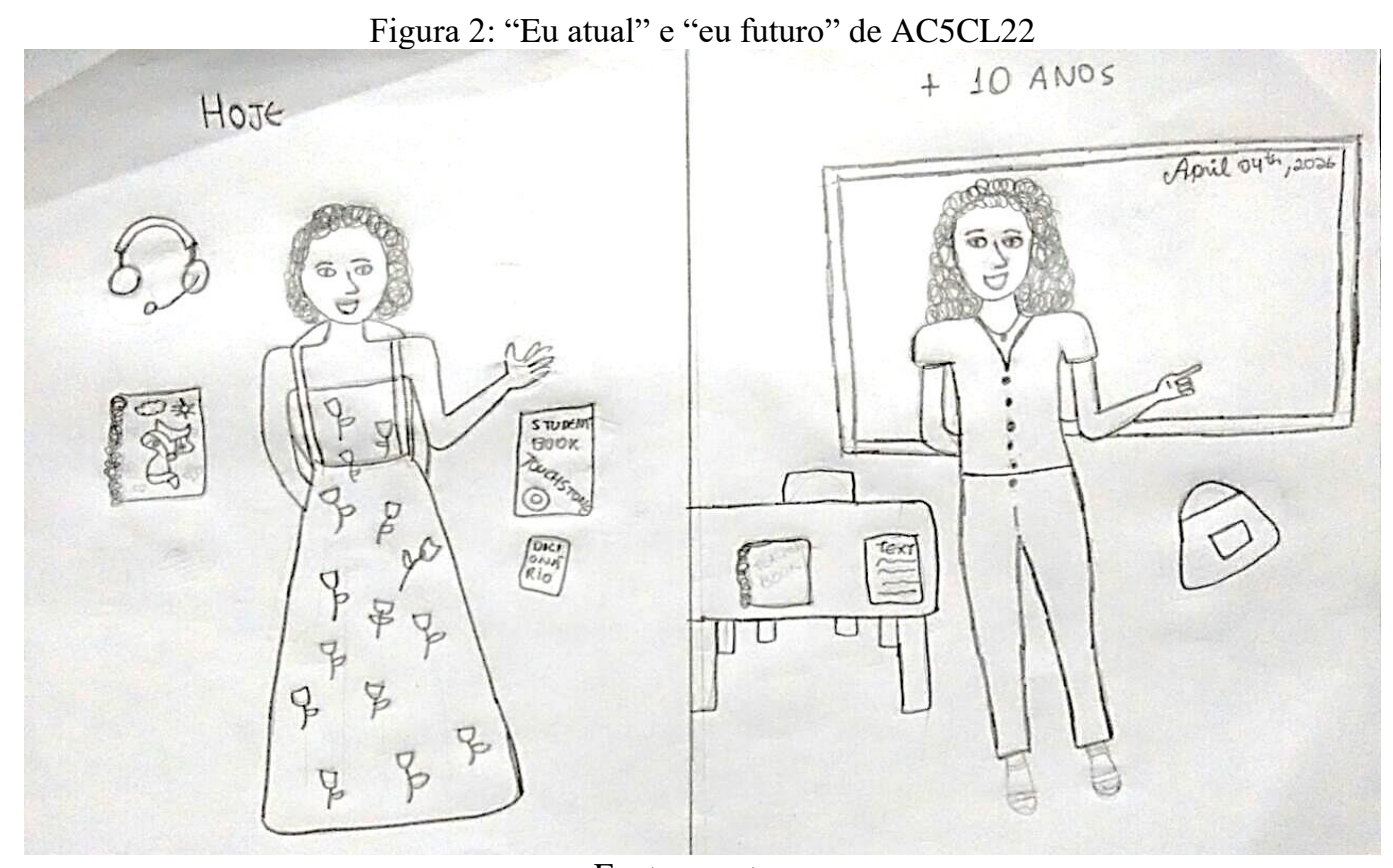

Fonte: a autora.

Em relação a isso, Little (1999, p. 77) relata que aprendentes são autônomos quando “[...] estabelecem seus próprios horários de estudo e se responsabilizam por planejar, monitorar e avaliar atividades particulares de aprendizagem, assim como o processo de aprendizagem como um todo"1.

\footnotetext{
${ }^{1}$ Minha tradução do original: "they set their own learning agenda and take responsibility for planning, monitoring and evaluating particular learning activities and the learning process overall” (LITTLE, 1999, p. 77).
} 


\section{CONCLUSÃO}

A leitura do modelo de Mynard (2012) ofereceu um parâmetro da prática do aconselhamento linguageiro. A observação da trajetória de aprendizagem da AC5CL22 e de suas práticas autônomas foi essencial para compreender que o aconselhamento pode ser, quando bem estruturado, uma peçachave na aprendizagem do aluno.

Por fim, tornou-se claro que o processo de aconselhamento deve viabilizar uma reflexão sobre o papel humano do aprendente. Esse deve compilar experiências positivas e propiciar um ambiente favorável à troca de informações com o conselheiro. A partir do momento em que o aconselhamento linguageiro é visto como algo positivo pelo aconselhando, essa prática se torna uma forma de alavancar o desenvolvimento de comportamentos autônomos que corroborem uma aprendizagem de línguas exitosa. 


\section{REFERÊNCIAS}

AOKI, N. Can do statements for advisors. In: LUDWIG, C. MYNARD, J. Autonomy in language learning: Advising in action. Kent: IATEFL, 2012. cap. 1, p. 154-163.

BENSON, P. Teaching and Researching Autonomy in Language Learning. Harlow: Pearson, 2001.

CARSON, L.; MYNARD, J. Introduction. In: MYNARD, J.; CARSON, L. Advising in Language Learning: dialogue, tools and context. Harlow: Pearson, 2012. cap. 1, p. 3-25.

ESCH, E. Promoting learner autonomy: criteria for the selection of appropriate methods. In: PEMBERTON, R. et al. Taking Control: Autonomy in Language Learning. Hong Kong: Hong Kong University Press, 1996. cap. 3, p.35-48.

GARDNER, D.; MILlER, L. Counselling. In: GARDNER, D.; MILLER, L. Establishing SelfAccess: from theory to practice. Cambridge: Cambridge, 1999. cap. 10, p. 180-203.

KATO, S. Professional development for learning advisors: Facilitating the intentional reflective dialogue. Studies in Self-Access Learning Journal, Chiba, v. 3, n. 1, p. 74-92, 2012.

LITTLE, D. Learner Autonomy: Definitions, Issues and Problems. Dublin: Authentik, 1991.

MOZZON-McPHERSON, M. The language adviser: A new type of teacher. An analysis of an emerging role. In: LITTLE, D.; VOSS, B. (Eds.). Language centres: Planning for the new millennium. Plymouth: Cercles, 1997. p. 97-109.

MOZZON-McPHERSON, M. Supporting Independent Learning Environments: An analysis of structures and roles of language learning advisers. System, Londres, v. 35, n. 1, p. 66-92, 2007.

MYNARD, J.; CARSON, L. (Ed.) Advising in Language Learning: dialogue, tools and context. Harlow: Pearson, 2012.

OXFORD, R. Language Learning Strategies: what every teacher should know. Heinle \& Heinle. Boston, 1990.

REINDERS, H. The what, why and how of language advising. MexTESOL, v. 32, n. 2, 2008, p. 1322. 